S. SASAO

KODAI MATH. J.

11 (1988), 306-315

\title{
ON THE HOMOTOPY OF CERTAIN MAPPING SPACES
}

Dedicated to Professor Hiroshi Toda on his 60th birthday

\author{
By SeIya Sasao
}

\section{$\S 0$. Introduction.}

We denote by map $(X, Y)$ the space of continuous maps $X \rightarrow Y$ preserving base points endowed with Compact-Open topology. We are interested in studing homotopy groups $\pi_{k}(\operatorname{map}(X, Y), f)$. This problem has been attacked in papers $[1],[2],[3],[5]$, and etc.. In this note we are mainly concerned with the subspace $\varepsilon(X, Y)$ consisting of homotopy equivalences.

Since $\pi_{k}(\varepsilon(X, Y), f)$ is isomorphic to $\pi_{k}\left(\varepsilon(X, X), 1_{X}\right)$ for any $f$ the study of $\pi_{k}\left(\varepsilon(X, X), 1_{X}\right)$, i. e. $\pi_{k}\left(\operatorname{map}(X, X), 1_{X}\right)$ is essential. Our purpose is to describe these homotopy groups in the case of $X$ being a principal bundle over a $n$ sphere $S^{n}$. One of difficulty for determing $\pi_{k}(\operatorname{map}(X, Y), f)$ arises from a choise of the base point. For example if we chose the trivial map as the base point these groups are explained as a set $\left\{\Sigma^{k} X, Y\right\}$, i. e. the group of homotopy classes of maps from the itterated suspension $\Sigma^{k} X$ to $Y$ preserving base points. Therefore, in this case, we consider the problem solved, so our purpose is in getting expression like this for our homotopy groups.

Let $p ; X \rightarrow S^{n}$ be a principal $G$-bundle. Then we have a fibre space

$$
\operatorname{map}(X, G) \longrightarrow \operatorname{map}(X, X) \longrightarrow \operatorname{map}\left(X, S^{n}\right),
$$

and therefore a long exact sequence:

$$
\begin{aligned}
& \pi_{k+1}\left(\operatorname{map}\left(X, S^{n}\right), p\right) \underset{\partial}{\longrightarrow} \pi_{k}\left(\operatorname{map}(X, G), \bar{e}_{0}\right) \\
& \quad \longrightarrow \pi_{k}\left(\operatorname{map}(X, X), 1_{X}\right) \longrightarrow \pi_{k}\left(\operatorname{map}\left(X, S^{n}\right), p\right)
\end{aligned}
$$

where $\bar{e}_{0}$ denote the constant $\operatorname{map} \bar{e}_{0}(X)=e_{0}$; the unit of $G$.

Hence we want to describe the boundary homomorphism

$$
\partial_{k+1}: \pi_{k+1}\left(\operatorname{map}\left(X, S^{n}\right), p\right) \longrightarrow \pi_{k}\left(\operatorname{map}(X, G), \bar{e}_{0}\right) .
$$

Of course, this needs some description of three objects:

$$
\pi_{k+1}\left(\operatorname{map}\left(X, S^{n}\right), p\right), \quad \pi_{k}\left(\operatorname{map}(X, G), \bar{e}_{0}\right) \text { and } \partial_{k+1} .
$$

Received April 8, 1988 
Let $\xi: S^{n} \rightarrow B_{G}$ be the characteristic map of the bundle. Then $\partial_{k+1}$ is equivalent to the induced homomorphism

$$
\xi_{*}: \pi_{k+1}\left(\operatorname{map}\left(X, S^{n}\right), p\right) \longrightarrow \pi_{k+1}\left(\operatorname{map}\left(X, B_{G}\right), \xi p\right),
$$

and the target of $\xi_{*}$ is isomorphic to the group $\left\{\sum^{k+1} X, B_{G}\right\}$ because of the contractibility of $\xi p$. However, in general, we could not have a suitable description of $\pi_{k+1}\left(\operatorname{map}\left(X, S^{n}\right), p\right)$ compatible with the above identification. On the other hand, we noted that the space $X / G$ which is obtained from $X$ by collapsing $G$ to the base point is homeomorphic to a wedge sum $S^{n} \vee \Sigma^{n} G$, and as a fundamental lemma, we will show in $\S 1$ that there exists an isomorphism:

$$
\pi_{k+1}(\operatorname{map}(X / G, Y), f) \cong \pi_{k+n+1}(Y)+\left\{\sum^{k+n+1} G, Y\right\}
$$

having convenient properties for describing $\partial_{k+1}$.

Thus we can determine $\partial_{k+1}$ for elements contained in the image:

$$
\pi_{k+1}\left(\operatorname{map}\left(X / G, S^{n}\right), \bar{p}\right) \longrightarrow \pi_{k+1}\left(\operatorname{map}\left(X, S^{n}\right), p\right),
$$

and moreover this is enable us to calculate homotopy groups of certain subspaces of $\operatorname{map}(X, X)$ (Theorem 2.3 and 2.4).

Here we give an example. Let $p: S^{7} \rightarrow S^{3}$ be the Hopf bundle and map $\left(S^{7} ; S^{2}\right)$ be the space of maps $\left(S^{7}, S^{3}\right) \rightarrow\left(S^{7}, S^{3}\right)$. Then we have

$$
\pi_{k}\left(\operatorname{map}\left(S^{7} ; S^{3}\right), 1\right) \cong \pi_{k+4}\left(S^{7}\right)+\pi_{k+7}\left(S^{7}\right)+\pi_{k+3}\left(S^{3}\right)
$$

although we have $\pi_{k}\left(\operatorname{map}\left(S^{7}, S^{7}\right), 1\right) \cong \pi_{k+7}\left(S^{7}\right)$.

Through out this paper we denote by $\infty$ the base point and by $\bar{\infty}$ the constant map $X \rightarrow \infty$ for any $X$.

\section{§1. A fundamental isomorphism.}

Let $X$ be the space $A \cup D^{n} \times A$ obtained from the following identification:

$$
(x, a) \equiv \xi(x, a), \quad \xi: S^{n-1} \times A \longrightarrow A, \quad \xi(\infty, a)=a,
$$

and let $X / A$ be the space obtained from collapsing $A$ to $\infty$. In this section we are mainly concerned with homotopy groups

$$
\pi_{*}(\operatorname{map}(X / A, Y), f) .
$$

First we note that there are the natural homeomorphism

$$
j:\left(D^{n} \times A / S^{n-1} \times A, \infty\right) \longrightarrow(X / A, \infty)
$$

and its induced homeomorphism

$$
j_{Y}:(\operatorname{map}(X / A, Y), f) \longrightarrow\left(\operatorname{map}\left(D^{n} \times A / S^{n-1} \times A, Y\right), f j\right) .
$$


Hence our p'urpose is to study $\pi_{*}\left(\operatorname{map}\left(D^{n} \times A / S^{n-1} \times A, Y\right), f j\right)$. Consider the fibre space

$$
r: \operatorname{map}\left(D^{n} \times A, Y\right) \longrightarrow \operatorname{map}\left(S^{n-1} \times A, Y\right)
$$

defined by restricting the domain of maps on $S^{n-1} \times A$. Since the fibre $r^{-1}(\infty)$ is just considered as the space $\operatorname{map}\left(D^{n} \times A / S^{n-1} \times A, Y\right)$ we have a long exact sequence:

$$
\begin{aligned}
\longrightarrow \pi_{*}\left(\operatorname{map}\left(S^{n-1} \times A, Y\right), \infty\right) & \longrightarrow \pi_{*}\left(\operatorname{map}\left(D^{n} \times A / S^{n-1} \times A, Y\right), f j\right) \\
& \longrightarrow \pi_{*}\left(\operatorname{map}\left(D^{n} \times A, Y\right), f j^{\prime}\right) \longrightarrow .
\end{aligned}
$$

On the other hand we have a commutative diagram

$$
\begin{gathered}
\left.\pi_{*}\left(\operatorname{map}\left(D^{n} \times A, Y\right), f j\right) \longrightarrow \pi_{*}\left(S^{n-1} \times A, Y\right), \bar{\infty}\right) \\
\pi_{*}(\operatorname{map}(\infty \times A, Y), \bar{\infty})
\end{gathered}
$$

where arrows denote homomorphism induced by maps analogus to the map $r$. Since it follows from the contractibility of the cell that the vertical arrow is isomorphic we can know the injectivity of $r_{*}$.

Hence from the long exact sequence we have

LEMMA 1.1. There exists an isomorphism

$$
\begin{aligned}
& \pi_{*}\left(\operatorname{map}\left(D^{n} \times A / S^{n-1} \times A, Y\right), f j\right) \\
& \quad \cong \pi_{*+1}\left(\operatorname{map}\left(S^{n-1} \times A, Y\right), \bar{\infty}\right) / \operatorname{proj}_{*} \pi_{*+1}(\operatorname{map}(A, Y), \bar{\infty}) .
\end{aligned}
$$

And moreover we have the standard isomorphism

$$
\left\{\Sigma^{*+1}\left(S^{n-1} \times A\right), Y\right\} \cong\left\{\Sigma^{*+1} S^{n-1}, Y\right\}+\left\{\Sigma^{*+1} A, Y\right\}+\left\{\Sigma^{*}\left(S^{n-1} \sharp A\right), Y\right\}
$$

where $S^{n-1} \# A$ denotes the reduced join of $S^{n-1}$ with $A$.

Now combining these isomorphisms we have an isomorphism

$$
\sigma_{Y}: \pi_{*}(\operatorname{map}(X / A, Y), f) \cong\left\{\Sigma^{*+1} S^{n-1}, Y\right\}+\left\{\Sigma^{*}\left(S^{n-1} \sharp A\right), Y\right\}
$$

Thus our fundamental lemma is the following

LEMMA 1.2. (1) $\sigma_{Y}$ is natural, i.e. for a map $\alpha: Y \rightarrow Z$ we have the commutative diagram

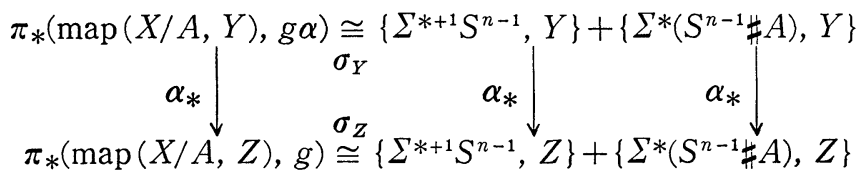

(2) Let us consider the fibring 


$$
\operatorname{map}(X / A, Y) \longrightarrow \operatorname{map}(X, Y) \longrightarrow \operatorname{map}(A, Y) .
$$

Then there exists the following identification of the boundary

$$
\begin{gathered}
\pi_{*+1}(\operatorname{map}(A, Y), \bar{\infty}) \longrightarrow \pi_{*}(\operatorname{map}(X / A, Y), f) \\
\left\{\Sigma^{*+1} A, Y\right\} \longrightarrow\left\{\Sigma^{*+1} S^{n-1}, Y\right\}+\left\{\Sigma^{*}\left(S^{n-1} \# A\right), Y\right\} \\
\beta \longrightarrow \beta \Sigma^{*+1} \lambda_{\xi}+\beta \Sigma^{*} c(\xi)
\end{gathered}
$$

where $\lambda_{\xi}=\xi \mid S^{n-1} \times \infty$ and $c(\xi)$ denotes the Hopf construction of $\xi$.

Proof. (1) easily follows from definitions. Next consider the commutative diagram of fibrings

$$
\begin{gathered}
\operatorname{map}(X / A, Y) \longrightarrow \operatorname{map}(X, Y) \longrightarrow \operatorname{map}(A, Y) \\
\operatorname{map}\left(D^{n} \times A / S^{n-1} \times A, Y\right) \longrightarrow \operatorname{map}\left(D^{n} \times A, Y\right) \longrightarrow \operatorname{map}\left(S^{n-1} \times A, Y\right) .
\end{gathered}
$$

Then, back to Lemma 1.1 and using the commutativity of the homotopy exact sequences the proof is completed from expressing

$$
\Sigma \xi: \Sigma\left(S^{n-1} \times A\right) \sim \Sigma S^{n-1} \vee \Sigma A \vee S^{n-1} \sharp A \longrightarrow \Sigma A
$$

as $\left(\Sigma \lambda_{\xi}, \Sigma i d, c(\xi)\right)$.

Here we give a few direct consequence of Lemma 1.2. Define a subgroup of $\left\{\Sigma^{*} A, Y\right\}$ by

$$
\Gamma_{*}(\xi: Y)=\left\{\alpha \mid \alpha \Sigma^{*} \lambda_{\xi}=0=\alpha \Sigma^{*-1} c(\xi)\right\}
$$

and a quotient group of $\left\{S^{*+n}, Y\right\}+\left\{\Sigma^{*+n} A, Y\right\}$ by

$$
\Delta_{*}(\xi: Y)=\left\{S^{*+n}, Y\right\}+\left\{\Sigma^{*+n} A, Y\right\} / \Delta\left\{\Sigma^{*+1} A, Y\right\}\left(\Sigma^{*+1} \lambda_{\xi}, \Sigma^{*} c(\xi)\right)
$$

where $\Delta$ is the diagonal map:

$$
\left\{\Sigma^{*+1} A, Y\right\} \longrightarrow\left\{\Sigma^{*+1} A, Y\right\}+\left\{\Sigma^{*+1} A, Y\right\}
$$

Proposition 1.3. If $Y$ is a topological group then there exists a short exact sequence

$$
\{0\} \longrightarrow \Delta_{*}(\xi: Y) \longrightarrow \pi_{*}(\operatorname{map}(X, Y), f) \longrightarrow \Gamma_{*}(\xi: Y) \longrightarrow\{0\} .
$$

Proof. Define a homeomorphism $\phi:(\operatorname{map}(X, Y), f) \rightarrow(\operatorname{map}(X, Y), \bar{\infty})$ by

$$
\phi(h)(x)=h(x) f(x)^{-1} .
$$

Since $\phi_{*}$ in $\pi_{*}$ is an isomorphism the proof follows from applying Lemma 1.2 to the case $f=\bar{\infty}$. 
Next, let $p$ be the map $X=A \cup D^{n} \times A \rightarrow S^{n}$ defined by

$$
p \mid A=\infty \text { and } p \mid D^{n} \times A=\left(D^{n} / S^{n-1}\right)\left(\text { proj. } D^{n}\right) .
$$

Since $p$ has a decomposition $X \rightarrow X / A \rightarrow S^{n}$ we have

Proposition 1.4. There exists a short exact sequence

$$
\{0\} \longrightarrow \Delta_{*}\left(\xi ; S^{n}\right) \longrightarrow \pi_{*}\left(\operatorname{map}\left(X, S^{n}\right), p\right) \longrightarrow \Gamma_{*}\left(\xi ; S^{n}\right) \longrightarrow\{0\} .
$$

For example, Let $X \rightarrow S^{n}$ be a $S^{m-1}$-bundle with its characteristic map $\xi: S^{n-1}$ $\rightarrow 0(m)$. Then $\Gamma_{*}\left(\xi ; S^{n}\right)$ and $\Delta_{*}\left(\xi ; S^{n}\right)$ can be described as follows:

and

$$
\Gamma_{*}\left(\xi ; S^{n}\right)=\left\{\alpha \mid \alpha \Sigma^{*} \lambda=0=\alpha \Sigma^{*-1} J(\xi)\right\} \subset \pi_{*+m-1}\left(S^{n}\right)
$$

$$
\Delta_{*}\left(\xi ; S^{n}\right)=\pi_{*+n}\left(S^{n}\right)+\pi_{*+n+m-1}\left(S^{n}\right) / \Delta \pi_{*+m}\left(S^{n}\right)\left(\Sigma^{*+1} \lambda, \Sigma^{*} J(\xi)\right)
$$

where $\lambda=\partial \iota_{n}$ is in the homotopy exact sequence of the bundle and $J$ denotes the $J$-homomorphism.

\section{§2. Principal $G$-bundles over $S^{n}$.}

Let $p: X \rightarrow S^{n}$ be a principal $G$-bundle with its characteristic map $\xi: S^{n} \rightarrow$ $B_{G}$. We are interested in homotopy groups

$$
\pi_{*}\left(\operatorname{map}(X, X), 1_{X}\right), \quad(* \geqq 0)
$$

where $\pi_{0}$ denotes a semi-group of homotopy classes of maps $X \rightarrow X$ with the distinguished element $1_{X}$.

Let $\tilde{p}: \operatorname{map}(X, X) \rightarrow \operatorname{map}\left(X, S^{n}\right)$ be the fibre space associated with the bundle and let the fibre $\tilde{p}^{-1}(p)$ identify with the $\operatorname{space} \operatorname{map}(X, G)$ which is considered as a subspace of map $(X, X)$ by imbedding $f(x) \rightarrow x \cdot f(x)$ where - denotes the right action of $G$ on $X$ as usual.

Then we have the homotopy exact sequence of the fibring:

$$
\begin{aligned}
& \longrightarrow \pi_{*}(\operatorname{map}(X, G), \bar{\infty}) \longrightarrow \pi_{*}\left(\operatorname{map}(X, X), 1_{X}\right) \\
& \longrightarrow \pi_{*}\left(\operatorname{map}\left(X, S^{n}\right), p\right) \longrightarrow \pi_{*-1}(\operatorname{map}(X, G), \bar{\infty}) \longrightarrow
\end{aligned}
$$

Here we note that $\operatorname{map}(X, G)$ naturally is a topological group and hence $\pi_{0}$ also is a group. However, in this case the boundary is homomorphic but the inclusion is not homomorphic. Now we want to determine the boundary

$$
\partial: \pi_{*+1}\left(\operatorname{map}\left(X, S^{n}\right), p\right) \longrightarrow \pi_{*}(\operatorname{map}(X, G), \bar{\infty}) \quad * \geqq 0
$$

The target can be identified with the group $\left\{\Sigma^{*} X, G\right\}$ but, in general, we have no expression like that about $\pi_{*}\left(\operatorname{map}\left(X, S^{n}\right), p\right)$ (see 1.4).

However, under some conditions, this is isomorphic to a quotient group of 
$\left\{S^{*+n}, S^{n}\right\}+\left\{\Sigma^{*+n} G, S^{n}\right\}$ so there is some posibility to calculate the boundary $\partial$. Now considering following diagrams of fibrings:
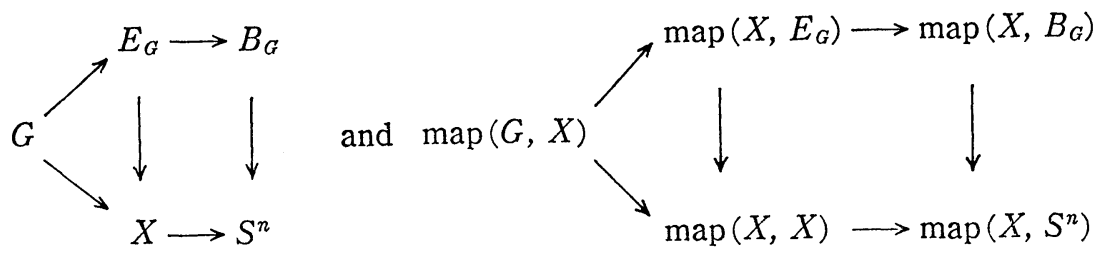

where upper fibrings denote universal fibrings for $G$ and $\operatorname{map}(G, X)$ we know that $\partial$ is equivalent to $\xi_{*}^{X}$, i. e. we have a commutative diagram

$$
\begin{gathered}
\pi_{*+1}\left(\operatorname{map}\left(X, S^{n}\right), p\right) \underset{\partial}{\longrightarrow} \pi_{*}(\operatorname{map}(G, X), \infty) \\
\pi_{*+1}\left(\operatorname{map}\left(X, B_{G}\right), \xi p\right) .
\end{gathered}
$$

Let $q: X \rightarrow X / G$ be the map collapsing $G$ to $\infty$. Then $q$ induces a diagram

$$
\begin{aligned}
& \pi_{*}\left(\operatorname{map}\left(X, S^{n}\right), p\right) \longrightarrow \pi_{*}\left(\operatorname{map}\left(X, B_{G}\right), \xi p\right) \\
& \uparrow q_{X / G *} \quad \uparrow q_{B_{G} *} \\
& \pi_{*}\left(\operatorname{map}\left(X / G, S^{n}\right), \bar{p}\right) \longrightarrow \pi_{*}\left(\operatorname{map}\left(X / G, B_{G}\right), \xi \bar{p}\right) .
\end{aligned}
$$

Next, let $\mu$ be the multiplication of $G$ and let $c(\mu): G \sharp G \rightarrow G$ be the Hopf construction of $\mu$. For any space $Y$, define a homomorphism

$$
\nabla_{*}(\xi: Y):\left\{\Sigma^{*+1} G, Y\right\} \longrightarrow\left\{S^{*+n}, Y\right\}+\left\{\Sigma^{*+n} G, Y\right\}
$$

by $\nabla_{*}(\xi: Y)(\alpha)=\alpha \Sigma^{*+1} \lambda_{\xi}+\Sigma^{*} \alpha c(\mu)\left(\lambda_{\xi} \# 1_{G}\right)$ where $\lambda_{\xi}=\partial \xi$ for the boundary $\partial: \pi_{n}\left(B_{G}\right)$ $\rightarrow \pi_{n-1}(G)$. Then, from Lemma 1.2 and above diagrams, we have

Proposition 2.1. Suppose that $q_{X / G^{*}+1}$ is onto. Then the kernel of

$$
\partial_{*+1}: \pi_{*+1}\left(\operatorname{map}\left(X, S^{n}\right), p\right) \longrightarrow \pi_{*}(\operatorname{map}(X, G), \bar{\infty})
$$

is isomorphic to a subgroup of $\pi_{*+n+1}\left(S^{n}\right)+\left\{\Sigma^{*+n+1} G, S^{n}\right\} / \nabla_{*+1}\left(\left\{\Sigma^{*+2} G, S^{n}\right\}\right)$, i.e. $\left(\xi_{*}\right)^{-1}\left\{\nabla_{*+1}\left(\xi: B_{G}\right)\left(\left\{\Sigma^{*+2} G, B_{G}\right\}\right) / \nabla_{*+1}\left(\left\{\Sigma^{*+2} G, S^{n}\right\}\right)\right.$.

Thus, since we have that $\pi_{*}\left(\operatorname{map}\left(G, S^{n}\right), \bar{\infty}\right)=0(*<n-\operatorname{dim} G)$ Proposition 2.1 implies

THEOREM 2.2. There exists a short exact sequence $(1 \leqq *<n-1-\operatorname{dim} G)$ : 


$$
\begin{aligned}
\{0\} & \longrightarrow\left(\xi_{*+1}\right)^{-1}\left(V_{*+1}\left(\xi: B_{G}\right) \text {-image }\right) \longrightarrow \pi_{*+n+1}\left(S^{n}\right)+\left\{\Sigma^{*+1+n} G, S^{n}\right\} \\
\longrightarrow & \left\{\Sigma^{*+1} X, B_{G}\right\} \longrightarrow \pi_{*}\left(\operatorname{map}(X, X), 1_{X}\right) \longrightarrow \partial_{*}^{-1}(0) \longrightarrow\{0\}
\end{aligned}
$$

Now we consider another fibrings. Let $\operatorname{map}(X ; G)$ be the subspace of $\operatorname{map}(X, X)$ consisting with maps preserving $G$ into $G$, and let Fib. $X$ be the subspace of maps preserving fibres. Clearly these spaces are related with each other through fibrings as shown in the following diagram

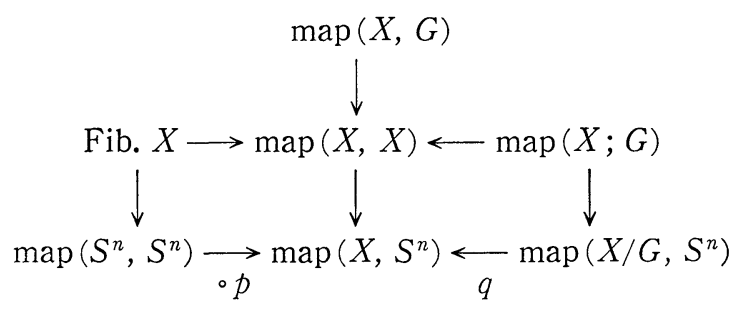

Using the commutativity of the diagram, first we obtain from Lemma 1.2

THEOREM 2.3. For $* \geqq 1$ there exist a short exact sequence:

$$
\begin{aligned}
\{0\} \longrightarrow\left(\xi_{*+1}\right)^{-1}\left(\nabla_{*+1}\left(\xi: B_{G}\right) \text {-image }\right) \longrightarrow \pi_{*+n+1}\left(S^{n}\right)+\left\{\Sigma^{*+n+1} G, S^{n}\right\} \\
\longrightarrow\left\{\Sigma^{*+1} X, B_{G}\right\} \longrightarrow \pi_{*}\left(\operatorname{map}(X ; G), 1_{X}\right) \\
\longrightarrow\left(\xi_{*}\right)^{-1}\left(\nabla_{*}\left(\xi ; B_{G}\right) \text {-image }\right) \longrightarrow\{0\}
\end{aligned}
$$

Secondly we investigate the fibring:

$$
\operatorname{map}(X, G) \longrightarrow \text { Fib. } X \longrightarrow \operatorname{map}\left(S^{n}, S^{n}\right) .
$$

We note that the fibre map $\circ p$ is decomposed as follows:

$$
\operatorname{map}\left(S^{n}, S^{n}\right) \longrightarrow \operatorname{map}\left(X / G, S^{n}\right) \longrightarrow \operatorname{map}\left(X, S^{n}\right)
$$

Let $\phi_{n}$ be a map: $\left(D^{n}, S^{n-1}\right) \rightarrow\left(S^{n}, \infty\right)$ of degree 1 . Since we may consider that $\pi_{*}\left(\operatorname{map}\left(S^{n}, S^{n}\right), 1_{S n}\right)$ is isomorphic to $\pi_{*}\left(\operatorname{map}\left(D^{n} / S^{n-1}, S^{n}\right), \phi_{n}\right)$ the homomorphism $(\circ p)_{*}$ may be considered as the (proj. $)_{*}$, i. e. we have a commutative diagram

$$
\begin{aligned}
& \pi_{*}\left(\operatorname{map}\left(D^{n} / S^{n-1}, S^{n}\right), \phi_{n}\right) \underset{\phi_{n_{*}}}{\longrightarrow} \pi_{*}\left(\operatorname{map}\left(S^{n}, S^{n}\right), 1_{S n}\right) \\
& \downarrow \text { (proj. })_{*} \quad \downarrow(\circ p)_{*} \\
& \pi_{*}\left(\operatorname{map}\left(D^{n} \times G / S^{n-1} \times G, S^{n}\right), \bar{\infty}\right) \underset{j_{*}}{\longrightarrow} \pi_{*}\left(\operatorname{map}\left(X / G, S^{n}\right), \bar{p}\right)
\end{aligned}
$$

Hence, considering the fibre map: 


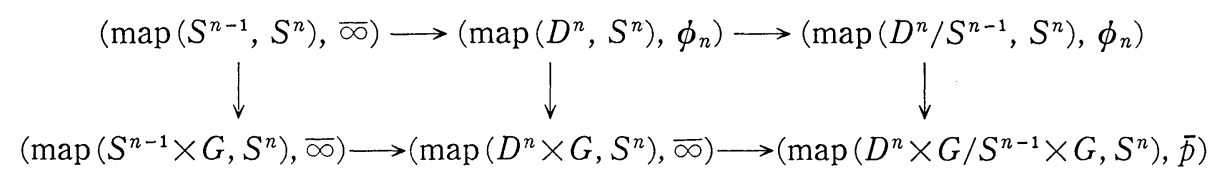

we have identifications:

$$
\begin{aligned}
& \begin{array}{c}
\pi_{*}\left(\operatorname{map}\left(X / G, S^{n}\right), \bar{p}\right) \underset{\bar{p}_{*}}{\longleftarrow} \pi_{*}\left(\operatorname{map}\left(S^{n}, S^{n}\right), 1_{S n}\right) \\
\cong \downarrow
\end{array} \\
& \pi_{*}\left(\operatorname{map}\left(D^{n} \times G / S^{n-1} \times G, S^{n}\right), \bar{p}\right) \underset{\operatorname{proj} \cdot *}{\longleftarrow} \pi_{*}\left(\operatorname{map}\left(D^{n} / S^{n-1}, S^{n}\right), \phi\right) \\
& \cong \downarrow \quad \operatorname{proj} * \text { } \cong \downarrow \\
& \left\{\Sigma^{*+1} S^{n-1}, S^{n}\right\}+\left\{\Sigma^{*+1}\left(S^{n-1} \sharp G\right), S^{n}\right\} \longrightarrow\left\{\Sigma^{*+1} S^{n-1}, S^{n}\right\}
\end{aligned}
$$

Now we define a subgroup $T_{*}(\xi)$ of $\pi_{*+n}\left(B_{G}\right)$ by

$$
T_{*}(\xi)=\left\{h \circ \Sigma^{*+1} \lambda_{\xi} \mid h \in\left\{\Sigma^{*+1} G, B_{G}\right\}, h \circ \Sigma^{*} c(\mu)\left(\lambda_{\xi} \# 1_{G}\right)=0\right\} .
$$

THEOREM 2.4. There exists a short exact sequence $\left(S_{*}(\xi)=\xi_{*}^{-1} T_{*}(\xi)\right)$ :

$\{0\} \longrightarrow \pi_{*+n+1}\left(S^{n}\right) / S_{*+1}(\xi) \longrightarrow\left\{\Sigma^{*+1} X, B_{G}\right\} \longrightarrow \pi_{*}\left(\right.$ Fib. $\left.X, 1_{X}\right) \longrightarrow S_{*}(\xi) \longrightarrow\{0\}$.

Proof. The proof is clearly completed from describ.ng the boundary

$$
\partial_{*}: \pi_{*}\left(\operatorname{map}\left(S^{n}, S^{n}\right), 1_{S n}\right) \longrightarrow \pi_{*-1}(\operatorname{map}(X, G), \bar{\infty})
$$

and this can be read off in the following diagram with Lemma 1.2.

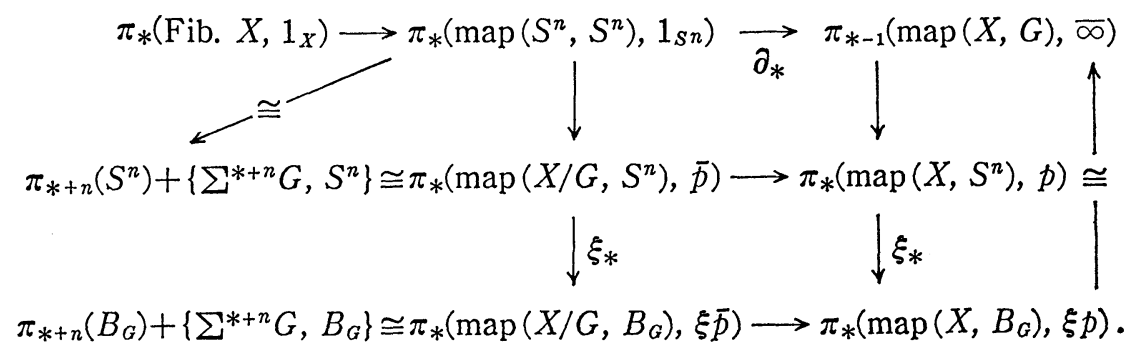

\section{§3. Examples.}

Let $p: X_{\xi} \rightarrow S^{n}$ be the $S^{3}$-principal bundle whose characteristic map is $\xi: S^{n}$ $\rightarrow B S^{3}$, and we call it a $N$-suspension trivial bundle if the $N$-fold suspension of $\partial \xi$ is trivial.

If $X_{\xi}$ is $N$-suspension trivial it is known in [4] that $\sum^{N-4} X$ has the homotopy type of $S^{N+n-4} \vee S^{N-1} \vee S^{N+n-1}$. Hence from Theorem 2.3 we have 
Example 3.1. If $p: X_{\xi} \rightarrow S^{n}$ is $N$-suspension trivial then there exists an exact sequence $(k \geqq N-1)$

$$
\{0\} \longrightarrow \xi_{k+1}^{-1}(0) \longrightarrow \underset{\pi_{k+n+4}\left(S^{n}\right)}{\pi_{k+n+1}\left(S^{n}\right)} \begin{gathered}
\pi_{k+n+1}\left(B S^{3}\right) \\
\pi_{k+n+4}\left(B S^{3}\right) \\
+ \\
\pi_{k+4}\left(B S^{3}\right)
\end{gathered} \longrightarrow \pi_{k}\left(\operatorname{map}\left(X_{m} ; S^{3}\right), 1\right) \longrightarrow \xi_{k}^{-1}(0) \rightarrow\{0\}
$$

where $\xi_{*}$ is the induced homomorphism by $\xi$, i.e.

$$
\xi_{*}: \pi_{*+n}\left(S^{n}\right)+\pi_{*+n+3}\left(S^{n}\right) \longrightarrow \pi_{*+n}\left(B S^{3}\right)+\pi_{*+n+3}\left(B S^{3}\right) .
$$

Remark. In the case $N=1$ we have $\xi=0$.

Next, let $p_{m}: X_{m} \rightarrow S^{2}$ be the $S^{1}$-principal bundle with its characteristic map $S^{1} \rightarrow S^{1}$ of degree $m$. Since $\left\{\Sigma^{*+1} X_{m}, B S^{1}\right\}=0=\pi_{*+2}\left(B S^{1}\right)$ for $* \geqq 1$ Theorem 2.4 gives

Example 3.2. $\pi_{*}\left(\right.$ Fib. $\left.X_{m}, 1_{X_{m}}\right) \cong \pi_{*+2}\left(S^{2}\right)(* \geqq 1)$.

At last, considering the $S^{1}$-principal bundle $S O(3) \rightarrow S^{2}$, Proposition 1.3 gives

Example 3.3. There exists a short exact sequence

$$
\{0\} \longrightarrow H(*, \eta) \longrightarrow \pi_{*}(\operatorname{map}(S O(3), S O(3)), 1) \longrightarrow G(*, \eta) \longrightarrow\{0\}
$$

where $H(*, \eta)$ and $G(*, \eta)$ are defined respectively as follows:

$$
\begin{aligned}
& H(*, \eta)=\pi_{*+2}\left(S^{2}\right)+\pi_{n+*+1}\left(S^{2}\right) /\left\{2 \alpha+\alpha \Sigma \eta, \alpha \pi_{*+2}\left(S^{2}\right)\right\} \\
& G(*, \eta)=\left\{2 \alpha=0=\alpha \Sigma^{*-1} \eta\right\} \pi_{*+1}\left(S^{2}\right) .
\end{aligned}
$$

Let $p: S^{7} \rightarrow S^{3}$ be the Hopf bundle, in which we have $\partial \hat{\xi}=\lambda_{\hat{\xi}}=\iota: S^{3} \rightarrow S^{3}$. Hence the followings follows from Theorem 2.3 and 2.4 respectively.

Example 3.4.

$$
\pi_{*}\left(\operatorname{map}\left(S^{7} ; S^{3}\right), 1_{S^{7}}\right) \cong \pi_{*+4}\left(S^{7}\right)+\pi_{*+7}\left(S^{7}\right)+\pi_{*+3}\left(S^{3}\right)
$$

Example 3.5. For the Hopf map $\nu: S^{7} \rightarrow S^{3}$ we define the homomorphism

$$
\left(\Sigma^{*} \nu\right): \pi_{*+4}\left(S^{3}\right) \longrightarrow \pi_{*+7}\left(S^{3}\right)
$$

by $\left(\Sigma *_{\nu}\right)(\alpha)=\alpha \Sigma *_{\nu}$. Then we have an exact sequence

$$
\begin{aligned}
\{0\} \longrightarrow \pi_{*+4}\left(S^{3}\right) /\left(\Sigma^{*} \nu\right)^{-1}(0) & \longrightarrow \pi_{*+7}\left(S^{3}\right) \longrightarrow \pi_{*}\left(\text { Fib. } S^{7}, 1\right) \\
& \longrightarrow \pi_{*+4}\left(S^{7}\right)+\left(\Sigma^{*-1} \nu\right)^{-1}(0) \longrightarrow\{0\} .
\end{aligned}
$$




\section{REFERENCES}

[1] H. Federer, A study of function spaces by spectral sequences, Trans. Amer. Math. Soc., 82 (1956), 340-361.

[2] M.G. BarratT, Track groups I, Proc. London Math. Soc., (3) 5 (1955), 71-106.

[3] J.M. Møller, On spaces of maps between complex projective spaces, Proc. Amer. Math. Soc., 91 (1984), 471-476.

- 4 S. SASAO, The stable group of self-homotopy equivalences of sphere bundles over the sphere, Kodai Math. Jour., 4 (1981), 231-238.

[5] S. SASAO, The homotopy of $\operatorname{Map}\left(C P^{m}, C P^{n}\right)$, Jour. London Math. Soc., (2) 8 (1974), 193-197.

[6] S.T. Hu, Concerning the homotopy groups of the component of the mapping space $Y^{S}$, Indag. Math., 8 (1946), 623-629.

[7] V.L. HANSEN, On spaces of maps of $n$-manifolds into the $n$-sphere, Trans. Amer. Math. Soc., 265 (1981), 273-281.

[8] G.W. WhitwheAd, A generalization of Hopf invariant, Ann. of Math., 51 (1950), 192-237.

Department of Mathematics

TOKYO INSTITUTE OF TECHNOLOGY

OH-OKAYAMA, MEgURO-KU

TOKYO, JAPAN 
